ESAIM: PROCEEDINGS, June 2008, Vol. 23, p. 30-47

L. Boudin, C. Grandmont, Y. Maday, B. Maury, B. Sapoval \& J.-F. Gerbeau, Editors

\title{
IMPACT OF RESPIRATORY MECHANICS MODEL PARAMETERS ON GAS EXCHANGE EFFICIENCY $* * *$
}

\author{
S. Martin ${ }^{1}$, T. Similowski ${ }^{2}$, C. Straus ${ }^{2}$ And B. Maury ${ }^{1}$
}

\begin{abstract}
In this article, we are interested in the impact of the mechanical characteristics of the bronchial tree and lung parenchyma on the gas exchange capacity of the human lungs.

Objectives. The precise role of the airway smooth muscle (ASM) remains a matter of debate. ASM could have a structural function, to confer transport optimized mechanical properties to the bronchial tree. To test this hypothesis, the present model analysis investigates how ASM-dependent changes in respiratory mechanics can influence the efficiency of the lung as gas exchangers.

Method. To this aim, two models have been elaborated. The first one is a single compartment model of the respiratory system that is accurate enough to predict the behaviour of the system during a complex dynamical situation (the forced inspiratory-expiratory maneuver that is performed during spirometric evaluations). The second one is an oxygen absorption model. The two models are coupled in series. The effects of changes in the parameters of the mechanical model on the performance of the gas exchange model are described.

Results. The model shows that the mechanical response of the bronchial tree to the dilating forces related to inspiration is among the determinants of this efficiency. At one extreme, a fully rigid bronchial tree would be counterproductive by preventing sufficient amounts of gas to flow through it and reach the alveoli. At the other extreme, a fully distensible bronchial tree would allow greater inspiratory volumes to enter the thorax, but would compromise gas exchange efficiency by decreasing the alveolar volume, hence the membrane surface available for oxygen transfer (deadspace effect). This supports the hypothesis that ASM has a transport optimizing structural function.

Conclusions. This is supported by physiological observations compatible with deadspace increases following the administration of ASM-relaxing agents, i. e. bronchodilators.
\end{abstract}

\footnotetext{
* The authors would like to thank C. Grandmont for the many interesting discussions on the subject.

** This work has been partially supported by the ANR project "Transport et Transfert via le Système Pulmonaire Humain" and by the ACINIM project "LePoumonVousDisJe".

${ }^{1}$ Université Paris-Sud XI, Laboratoire de Mathématiques - CNRS-UMR 8628, Bât. 425, 91405 Orsay Cedex, France

${ }^{2}$ UPRES EA 2397, Université Paris VI Pierre et Marie Curie, Paris, France
}

(c) EDP Sciences, SMAI 2008 
Résumé. Dans cet article, nous nous intéressons à l'impact des caractéristiques mécaniques de l'arbre bronchique et du parenchyme pulmonaire sur les échanges gazeux dans le poumon humain.

Objectifs. Le rôle du muscle lisse bronchique est imparfaitement compris. Ce rôle pourrait être en partie structurel, pour conférer à l'arbre bronchique des caractéristiques mécaniques compatibles avec un transport optimal de l'air inspiré vers les zones d'échange.

Méthode. Pour tester cette hypothèse, deux modèles ont été élaborés. Le premier est un modèle mono-compartimental du système respiratoire permettant de prévoir le comportement du système durant une situation dynamique complexe (les manoeuvres inspiratoire et expiratoire forcées qui sont réalisées lors d'examens spirométriques). Le second est un modèle d'absorption de l'oxygène. Les deux modèles sont couplés en série. Les effets de modifications des paramètres du modèle mécanique sur le modèle d'absorption sont décrits.

Résultats. Le modèle montre que la réponse mécanique de l'arbre bronchique aux forces de dilatation relatives à l'inspiration joue un rôle déterminant dans l'efficacité respiratoire. A un extrême, un arbre bronchique totalement rigide serait contre-productif en empêchant une quantité suffisante de gaz d'atteindre les alvéoles. A l'autre extrême, un arbre bronchique totalement déformable permettrait à de très grands volumes d'air d'entrer dans le thorax mais compromettrait l'efficacité des échanges gazeux en limitant le volume alvéolaire et donc la surface de membrane disponible pour le transfert d'oxygène (effet "espace mort"). Ces observations sont en faveur d'une hypothèse du rôle structurel du muscle lisse bronchique destiné à optimiser les propriétés convectives de l'arbre bronchique.

Conclusions. Ces observations vont dans le sens de l'augmentation de l'espace mort qui peut suivre l'administration d'agents relaxant le muscle lisse bronchique, i. e. bronchodilatateurs.

\section{CONTEnts}

1. Modelling of the respiratory system: a short review

2. Mechanical lung model: a single-compartment model

2.1. Parameters and model

2.2. Numerical validation

3. Gas exchange in the lung model

3.1. Notion of efficiency: variations of the exchange surface area due to the airway wall compliance

3.2. $\mathrm{O}_{2}$ impoverishment

3.3. Numerical results: influence of the bronchial wall compliance

4. Conclusion

References

\section{Modelling of THE RESPiRAtory SYSTEM: A SHORT REVIEW}

Physiological modelling with mechanical and/or mathematical models has been a major focus in the field of bioengineering. Such models are useful in understanding physiological functions or processes, and can lead to the development of new diagnostic or therapeutic strategies. The modelling of the respiratory system, which has always been a subject of intensive studies, in constantly gaining interest, because of the increasing recognition of the complexity of the mechanisms at play during lung diseases. Modelling the respiratory system is difficult, owing to its great complexity. Indeed the respiratory systm is constituted of numerous elements of very different nature acting both in series and in parallel. These include a multidimensional rib cage, that is driven by an array of respiratory muscles answering to complex neural commands. Rib cag displacements provoke lung inflation and deflation, which produce intrathoracic pressures. These in turn drive inspired and expired gases through convective elements of complex geometry, that are linked to countless units where gas exchanges occur through 
membrane diffusion. To add to this intricacy, inspiration and expiration are highly asymmetrical processes. In humans at rest, inspiration is an active phenomenon depending on the contraction of inspiratory muscles, and it is somewhat self promoting, because as the lungs inflate the bronchial caliber increases and the resistance of the bronchial tree decreases. Conversely, expiration in humans at rest is driven solely by the elastic recoil of the lungs, and this is only during exercise or in response to an agression that the contraction of expiratory muscles supplements the elastic recoil. In contrast to inspiration, expiration is self-limiting because as the lung deflates the bronchial caliber decreases and the resistance of the bronchial tree increases. This intricacy explains that the dynamical behaviour of the respiratory system is complex and nonlinear, all the more so that it involves coupling effects with other systems such as circulation or locomotion. The mechanical behaviour of the respiratory system reflects the physical properties of its intrinsic components. Therefore, it can be described through the relationships that exist between observable quantities, such as pressures, flows, and volumes.

A huge number of mathematical models of the respiratory system have been proposed. From 0D models to $3 \mathrm{D}$ models, the lung is often represented as a multicompartment system in which one may include specific coupling systems. The level of details in the modelling highly depends on the investigated quantities and of the nature of the pathological application that is considered. Some of the models limit themselves to particular aspects of the respiratory system, whereas others aim at describing the system globally. These models do not however necessarily focus on crucial aspects of respiratory physiology, such as the gas flow to and from the lung, the mixing of gas inside the lung, the gas exchange between lung and the blood, the blood gas transport system etc.

In this study, instead of working with a fully detailed lung model with a great number of degrees of freedom, we aim at describing a simple model which would mimic the mechanical behaviour of the respiratory system, at least when focusing on observable quantities such as flows, volumes, pressures, oxygen concentration, carbone dioxyde concentration, and which would give access to a better understanding of the influence of the characteristics of the bronchial convection system on gas exchanges themselves.

\section{Single-compartment models}

The simplest model of the respiratory system is assumed as a single-compartment model, often represented under the form of a balloon representing the lungs, connected to a pipe representing the airway. Inside this framework, many assumptions can be formulated, depending on the level of required precision (we may refer to [5] for a brief description of such models and their connections). Let us mention that, in [5], a hierarchy of different mathematical models (at different scales and different degrees of complexity) are presented. All the models describe the lung as a single container of air and focus on $P-V$ relationships for both static [4,7,24] and dynamic regimes [1]. Let us sketch the basic principle which leads to these models. In the dynamic setting, when dealing with a flexible lung, one may assume that pressure $P$ is related to a resistive pressure (trachea) and an elastic pressure (lung) as

$$
P(t)=E V(t)+R \dot{V}(t),
$$

where the so-called elastance $E$ and resistance $R$ are parameters to be identified (depending on the subjects, diseases, etc.). Fitting models to experimental data by least squares provide values for these parameters in some specific regimes. A way to make the models more realistic is to introduce nonlinear phenomena in $P-V$ laws: the model may be made nonlinear by making $R$ and $E$ depend on flow and volume, as will be proposed in our model. $R$ typically decreases when lung volume increases, and reciprocally; it also increases with airway diseases such as asthma or chronic obstructive pulmonary disease, as a result of an abnormal airway smooth muscle reactivity or of modified mechanical linkage between the bronchi and the parenchyma. $E$ is less sensitive than $R$ to lung volume and is roughly constant over the range of physiological volumes, except when lung inflation approaches its maximal value; it can depend on the dynamics of respiratory movements, and exhibits a marked frequency dependency. In disease, $E$ is typically increased by pathological processes such as lung fibrosis that rigidify the interstitial tissue, and decreased by pathological processes such as emphysema that rarefies structural components of the lungs and more specifically its elastic architecture. 
Some models, e.g. such as the ones described in [5], take into account the compressibility of air within the alveoli. Yet, the marginal pressures implied in the respiratory process are about 200 times smaller than the atmospheric pressure, which induces relative volume variations of the order of $0.5 \%$. We shall neglect this effect in the present approach.

As for inertance, this may play a significant role in some specific situations (such as high frequency forced maneuvers in normal subjects or obesity conditions) but it is known to be neglectible in standard regimes. In our model, inertance is taken into account even if it has no major influence on the system.

\section{Multi-compartment models}

The functional asymetry of the respiratory system has led many authors to investigate multi-compartment models. Moreover, the considerable interest in modelling low-frequency respiratory mechanics in terms of twocompartments is stated as it gives a much improved description of experimental data. Linear two-compartment models of the respiratory system have been proposed with two different types of gas redistribution throughout the lungs: the first one, which is called the parallel gas redistribution model, has dominated the literature since its introduction [17] and consists of a parallel arrangment of alveolar eparated compartments connected to the trachea; the second one, which is called the series gas redistribution model, is made of two balloons connected in series [16]. Starting from this kind of observations, numerous multi-compartment models can be described.

In [2], a model describing respiratory mechanics with the characteristics of two lung compartments including the viscoelastic properties is proposed. Of note, a two-compartment behaviour can be produced by different physiological mechanisms (e.g. gas distribution or tissue viscoelasticity [20]) between which discrimination requires additional inputs into the model; in particular, in [20], two classes of of two-compartment models that are compatible with pressure-flow relationships of air measured at the airway opening are investigated: one type of model accounts for regional ventilation inhomogeneity in the lung in terms of two alveolar compartments; the other type of model considers pulmonary ventilation to be homogeneous, while the tissues of the respiratory system are modelled as being viscoelastic. In [8], the dynamic behaviour of the two-compartment respiratory system in response to an arbitrary applied inspiratory pressure is investigated, providing output variables such as tidal volume, end-expiratory pressure, minute ventilation, mean alveolar pressure for each compartment. In [9], a five-compartment model is developped to simulate the effect of airway dynamics on ventilation distribution under different conditions. The model is to be incorporated into a particle lung deposition model to determine the nonuniformity of particle deposition in the lung, resulting from nonuniform ventilation distribution ${ }^{1}$.

In a general way, the choice of compartment model highly depends on the specific aspects of the respiratory system to be investigated. Notice that single-compartment models still make sense: studies with the alveolar capsule in dogs [6], mice [21] and other species showed that the normal lung ventilated at normal breathing frequencies is best described by a uniformly ventilated compartment surrounded by viscoelastic tissue.

Let us also mention that multi-compartment approaches, as carried out in $[10,11,15]$ have been recently proposed. Those approaches make it possible to include inertial effects on the proximal part of the tree, and provide an efficient tool to investigate the influence of asymmetry in the pressure distribution over the respiratory tree. Yet healthy symmetric lungs are known to behave like standard single-compartment 0D models.

\section{Gas exchange modelling}

Data from several different experiments suggest that complex interactions between the respiratory system and cardiac output exist in humans. Such interactions include the synchronisation between lung ventilation rate and the heart beat, and other examples. Mathematical models to describe the mechanisms underlying these interactions are being developped with the aim of improving our understanding of these physiological phenomena. Historically, gas exchange in the lungs has been modelled and studied at various different levels

\footnotetext{
${ }^{1}$ This model is based on the following observation: when the inspiratory flow rate is increased, the resistance of the airways begins to play a significant role in ventilation distribution. Experimental data show ventilation is more uniform at higher flow rates. Because airway resistance is changed by the physical properties of the inspired gas, ventilation is also altered when different gases are inspired.
} 
of detail. As the function of the lung is to bring $\mathrm{O}_{2}$ into the bloodstream, the purpose of a lung model is to replicate the connection between the atmosphere and the alveoli. In that prospect, we aim at defining an efficiency criterion related to the quantity of $\mathrm{O}_{2}$ that has been brought into the bloodstream during a few cycles. Therefore, the lung model must produce pressure-volume diagrams and keep track of the partial pressures of gases in expired air and in the alveoli.

In [5] a hierarchy of models of increasing complexity for gas exchange in the human lungs is presented: $(i)$ the first model represents the lung as an inflexible, single compartment; ( $i i)$ the second model represents the lung as a flexible, single compartment; $i i i$ ) the third model represents the lung as a flexible single-compartment with gas exchange; and $(i v)$ the final model represents the respiratory system as a flexible multi-compartmental model with gas exchange and gas transport. The models developped in [5] provide a mathematical framework which is able to link together molecular scale and whole systems scale models. Thus it appears that the proposed models apply in a general framework, depending on the quantities to be investigated.

In [23] a two-compartment model of lung is coupled to a reconstruction of alveolar gas composition: this allows to estimate non-invasively arterial carbon dioxide partial pressure, this quantity being useful to appreciate the efficiency of breathing and the control of breathing during exercise.

The single-compartment models of the lung assume a uniform alveolar gas: this allows one to consider there to be a uniform gas mix in the compartment, and thus to easily calculate the partial pressures of gases in the air mix. But when dealing with models involving multi-compartments, it is not so clear: in [18] (Chapter 8) a more complex model is used, partitioning the lung into several sections and describing an oscillatory gas flow in and out of these sections. The model takes the form of an equivalent electrical network, as the lung is supposed to be made of connected elementary subsystems. In particular, the lung model allows simulations with different compliances and air flow resistances and produces pressure-volume diagrams. This approach is combined with a model of a mix of gases in the lung sections, as both the partial pressures (for deciding the transport over the lung membrane) and the composition of the expired air for output are needed. For this, a pressure model, a gas composition model and a model of the gas mix from the overall flows are used.

\section{Organization of the paper}

In the present study, our aim is to develop a model that would allow a description of the role of airway smooth muscle state on gas exchanges. Indeed, changes in airway smooth muscle tone are bound to change the mechanical properties of the airway, their geometry, and their volume. These changes can in turn influence gas exchanges, for instance by changing the volume of inspired air available to reach the alveoli, by changing the regime of the gas flow entering them, or by changing the site of the transition between convection and diffusion mechanisms $[12,15,26]$. To this aim, we present a model for the respiratory system with one degree of freedom, denoted $x$, corresponding to the amplitude of the deformation of the thorax.

In Section 2, we present a nonlinear spring-mass-friction model:

- in Subsection 2.1, we briefly introduce a linear model; then, we introduce a nonlinear spring constant, based on critical volume variations of the rib cage; finally, we consider that the volume variation of the rib cage is divided between bronchi and alveoli, by introducing a weight parameter $\theta$ (which characterizes the intrinsic compliance of the bronchial wall). Based on assumptions related to the bronchial deformations, we express the resistance of the bronchial tree as a function of its volume so that the resistance evolves, during a breath cycle, with respect to $x$;

- Subsection 2.2 compares numerical curves predicted by the model to physiological spirometric curves obtained in humans during the so-called forced maneuvers.

In Section 3, we improve the model by focusing on gas exchanges considered as the main function of the respiratory system:

- in Subsection 3.1, in order to quantify the efficiency of the process, we explicitely introduce the quantity of $\mathrm{O}_{2}$ which has diffused into blood from some initial time;

- in Subsection 3.2, we investigate an improved model which takes into account the $\mathrm{O}_{2}$ impoverishment; 
- in Subsection 3.3, we focus on a parameter study with respect to the efficiency criterion and taking into account the complete model. More precisely, we investigate the influence of the airway wall compliance.

\section{MechanicAl LUnG MODEL: A SINGLE-COMPARTMENT MODEL}

\subsection{Parameters and model}

\section{Parameters}

Let us introduce the main parameters and variables leading to the modelling of the lung:

- $x$ (piston displacement) corresponds to the piston displacement relatively to the equilibrium position (at rest). Physiologically, it is related to the amplitude of the costal moves.

- $m$ (displaced mass) corresponds to the mass of the lung or, more precisely, the mass of the displaced set so that $m \dot{x}^{2} / 2$ corresponds to the kinetic energy that is involved.

- $k_{0}$ (spring constant) measures the stiffness related to stretching forces so that the lung comes back to rest spontaneously.

- $S$ (spring surface) corresponds to the effective surface on which parenchymal pressure applies.

- $\mu$ (tissue resistance) measures the resistive forces to deformation inside tissues so that $\mu \dot{x}$ is the instantaneous work dissipated in those tissues. Tissue resistance occurs due to friction between the tissue of the lung and the chest wall.

- $R_{0}$ (airway resistance) measures the resistives forces in all the bronchial tree so that

$$
\text { (external pressure) }-(\text { internal pressure })=R_{0} \times(\text { flow })
$$

The (atmospheric) external pressure is 0 and the internal pressure corresponds to the alveolar pressure. Airway resistance occurs due to the friction between the gas molecules and the walls of the airways.

- $f_{e}$ (external forces) corresponds to the muscular efforts leading to the deformation of the thoracic cage, which induces inspiration and expiration.

\section{Simplified model}

We denote by $p$ the alveolar pressure. As the flow is the time derivative of the volume, we have $-p=R_{0} S \dot{x}$. Moreover, this pressure applies on the spring with surface $S$, so that $m \ddot{x}=f_{e}-k_{0} x-\mu \dot{x}+S p$ and

$$
m \ddot{x}+\left(R_{0} S^{2}+\mu\right) \dot{x}+k_{0} x=f_{e} .
$$

This model may be represented in the framework of classical single-compartment models, as in FiG.1, for which the lung elastance, $E$, is equivalent to $k_{0} / S^{2}$, the lung resistance $R_{\ell}$ is equivalent to $\mu / S^{2}$ and the lung inertance, $I_{\ell}$, is equivalent to $\mathrm{m} / \mathrm{S}^{2}$. Notice that the effective resistance is made of two resistances in series $R_{0}$ and $R_{\ell}$.

This model does not take into account the surface tension at alveolar membranes. Nevertheless, the state at rest corresponds to a situation in which the negative pressure inside the parenchyma is equal to the pressure gap linked to the surface tension. This effect may be explicitely integrated to the model, which leads to a nonlinear model, but we can also consider that the initial model has been derived up to the parenchymal pressure, by including the effect of surface tension in the spring constant $k_{0}$.

In the forthcoming paragraphs, we state how it is possible to introduce nonlinear effects on the resistance $R$ and the spring constant $k$. For this, instead of Eq. (1), we will rather consider the following O.D.E

$$
m \ddot{x}+\left(R(x) S^{2}+\mu\right) \dot{x}+k(x) x=f,
$$

where $R$ and $k$ have to be precised. 


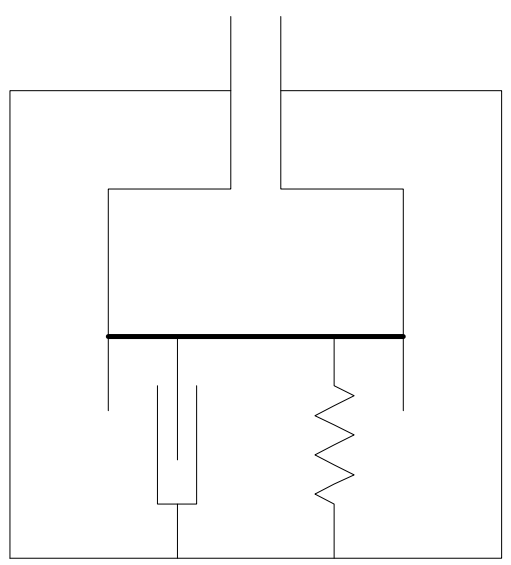

Figure 1. The lung modeled as a flexible container. $V=S\left(x_{0}+x\right)$ : total lung volume, $R$ : overall resistance of the conducting airways, $k$ : spring constant, $\mu$ : friction coefficient. The moving plate has mass $m$ and area $S$.

\section{Variations of the spring constant due to the critical volume constraints}

In the case of forced maneuvers at deep inspiration (resp. expiration), the rib cage volume obviously cannot exceed some upper (resp. lower) critical size: this can be modelled by taking into account a nonlinear spring constant. We denote $\delta V_{\max }=S x_{\max }>0$ (resp. $\delta V_{\min }=S x_{\min }<0$ ) the maximal (resp. minimal) lung volume variation which is determined by the experimental data (to be discussed further). During forced maneuvers (which allow the subject's lung to reach those critical volumes), we may assume that the spring constant is nonlinear, namely

$$
k(x):=k_{0}+k^{(e)}(x)+k^{(i)}(x) .
$$

In particular, $k^{(e)}$ (resp. $\left.k^{(i)}\right)$ is the spring contribution which is only active for negative (resp. positive) values of $x$, i. e. during expiration (resp. inspiration). We will assume that

$$
k^{(e)}(x):=\left\{\begin{array}{ll}
K_{e} x / x_{\min }, & \text { if } x<0 \\
0, & \text { if } x \geq 0
\end{array}, \quad k^{(i)}(x):= \begin{cases}0, & \text { if } x \leq 0 \\
K_{i} x / x_{\max }, & \text { if } x>0\end{cases}\right.
$$

where $K_{e}$ and $K_{i}$ are positive constants to be precised. Notice that the spring constant tends to increase linearly not only for positive increasing volumes but also negative decreasing volumes, thus preventing the lung volume from exceeding the critical values. Additionnally, the nonlinear contributions of the spring constant have only little influence for small volume variations. More precisely, the way to obtain the stabilization of the lung volume at critical values during forced maneuvers is obtained as follows: considering the behaviour of the lung during e.g. forced expiration (meaning that a maximal negative force $f_{e}=f_{\min }<0$ is imposed), as will be noticed on the experimental curves (to be further discussed) we have $\ddot{x}=\dot{x}=0$ and Eq. (1) reduces to

$$
k\left(x_{\min }\right) x_{\min }=f_{\min },
$$

which provides the relationship $K_{i}=f_{\min } / x_{\min }-k_{0}$. In the same way, now considering the behaviour of the lung during forced inspiration (meaning that a maximal positive force $f_{e}=f_{\max }>0$ is imposed), we have also $\ddot{x}=\dot{x}=0$ and Eq. (1) reduces to

$$
k\left(x_{\max }\right) x_{\max }=f_{\max },
$$

which provides the relationship $K_{e}=f_{\max } / x_{\max }-k_{0}$. Thus, Eq. (2) may be adapted with a nonlinear spring constant as

$$
k(x)=k_{0}+ \begin{cases}\left(f_{\min } / x_{\min }-k_{0}\right) x / x_{\min }, & \text { if } x<0 \\ \left(f_{\max } / x_{\max }-k_{0}\right) x / x_{\max }, & \text { if } x \geq 0\end{cases}
$$


Variations of the airway resistance due to the mechanical properties of the bronchial tree

We would like to include the influence of the smooth muscle which prevents some bronchi from distorting too much (in case of hyperactivity, it can even diminish the diameter of the bronchi). In the framework of the simple model (2), we may assume that the volume increase of the rib cage, which is equal to $S x$, divide between bronchi and alveoli, which is taken into account by a distribution parameter $\theta$. The total variation of volume $\delta V=S x$ is the sum of $\delta V_{A}$ (for the alveoli) and $\delta V_{B}$ (for the bronchi) with

$$
\delta V_{A}=(1-\theta) S x, \quad \delta V_{B}=\theta S x
$$

It is based on the conservation of the volume, assuming that the parenchyma tissues have a constant volume. In the sequel, we will denote $V_{A}^{0}$ (resp. $V_{B}^{0}$ ) the volume of the alveoli (resp. the bronchi) at rest. We will also denote $V_{A}$ (resp. $V_{B}$ ) the volume of the alveoli (resp. the bronchi) which is related to the previous quantities by the following relationships:

$$
V_{A}=V_{A}^{0}+(1-\theta) S x \quad\left(\operatorname{resp} . V_{B}=V_{B}^{0}+\theta S x\right)
$$

Remark 2.1. The parameter $\theta$ quantifies the action of the intrinsic compliance of the bronchial wall in the following sense: the so-called neutral value (or default value) of $\theta$ is associated to a similar deformation of bronchi and alveoli so that the volume variations are proportional to the initial volumes, respectively. Thus, one has

$$
\frac{\delta V_{A}}{V_{A}^{0}}=\frac{\delta V_{B}}{V_{B}^{0}}
$$

and this situation corresponds to the neutral value

$$
\theta_{0}=\frac{V_{A}^{0}}{V_{A}^{0}+V_{B}^{0}}
$$

A typical neutral value for human lungs is $\theta_{0} \approx 0.4$. The range $\theta<\theta_{0}$ may correspond to an effective activity of the smooth muscle: in particular, when $\theta$ is small, the activity of the smooth muscle may be considered as important since it tends to prevent the bronchial tree volume from varying.

We now assume, in a very simple manner ${ }^{2}$, that the bronchi are deformed without any modification of their shapes. As the resistance of a bronchus is proportional to $L / D^{4}$ ( $L$ and $D$ are respectively the length and diameter of the bronchus), for any given shape it is reciprocally proportional to the volume. We have

$$
R(x)=\frac{R_{0}}{1+\theta S x / V_{B}^{0}} .
$$

\subsection{Numerical validation}

In order to validate the model, we propose to compare numerical results to experimental ones, in a simple setting.

\section{Experimental description}

Experimental time-volume curves have been obtained using a classical bell spirometer (invented by John Hutchinson in 1852) and the flow has been computed using a time derivation. The procedure follows the following lines: a subject is first asked to breathe quietly, then to empty his or her lungs as much as possible,

\footnotetext{
${ }^{2}$ Our approach may be easily generalized by considering that the distortion of the bronchial tree follows some scenario (for instance, some bronchi deform with increasing volume while some bronchi deform with decreasing volume) which would be obtained from some information on elastance (linked to the presence, or not, of the smooth muscle, which has azimuthal or longitudinal orientation). This would lead to express the resistance as $R=1 / \Psi\left(V_{b}\right)$, where $\Psi$ is a non-decreasing function satisfying $\Psi(0)=0$ and plays the role of a shape-function.
} 
and then to breathe in up to the total lung capacity. From this point, after a brief pause, the subject is asked to forcibly expel the air contained in his or her thorax by blowing out as fast and as long as possible. The timevolume curve shown on Fig. 3 represents the volume variation $\delta V$ as a function of time. Thus, it corresponds to the graph of

$$
t \mapsto \delta V(t)
$$

As mentioned before, Fig. 3 allow us to define the upper critical volume variation $\delta V_{\max } \approx 2.50 \cdot 10^{-3} \mathrm{~m}^{3}$ and, in view of our single-compartment model, its corresponding upper critical displacement $x_{\max } \approx 0.20 \mathrm{~m}$. In the same way, we identify the lower critical volume variation $\delta V_{\min }=-2.50 \cdot 10^{-3} \mathrm{~m}^{3}$ and its corresponding lower critical displacement $x_{\min }=-0.25 \mathrm{~m}$. Forced Vital Capacity (FVC) is the amount of air that can be maximally forced out of the lungs after a maximal inspiration. For the tests to be of significance, it is recommended that the forced effort be 6 seconds or longer in duration. Forced Expired Volume in one second (FEV1) is the volume expired in the first second of maximal expiration after a deep inspiration and is a useful measure of how quickly the lungs can be emptied. FEV1/FVC gives a clinically useful index of bronchial obstruction that is defined by a disproportionate reduction of FEV1 as compared to FVC. In the presence of airflow obstruction, comparing FEV1 to the vital capacity measured during a complete but slow exhalation (slow vital capacity, SVC) from total lung capacity to residual volume - the volume beyond which it is impossible to further empty the lungs - shows an even more pronounced disproportion (FEV1/SVC smaller than FEV1/FVC). Notice also that Functional Residual Capacity (FRC) denotes the amount of air left in the lungs after a tidal breath out: it will be considered as the zero value for the time-volume curves.

Flow volume loops are two-dimensional phase portraits of the evolution of lung volume with time; they are perhaps the most recognizable of all pulmonary function tests. Clinicians can identify respiratory conditions simply by looking at the shape of the curve.

\section{Numerical description}

In order to reproduce the experimental curves observed in Fig. 3, we have used Eq. (2)-(3) with realistic coefficients:

$$
m=0.3 \mathrm{~kg}, \quad S=0.011 \mathrm{~m}^{2}, \quad k_{0}=36.3 \mathrm{~N} \cdot \mathrm{m}^{-1}, \quad R_{0}=1.33 \cdot 10^{5} \mathrm{~Pa} \cdot \mathrm{s} \cdot \mathrm{m}^{-3}, \quad \mu=4.02 \mathrm{~Pa} \cdot \mathrm{s} \cdot \mathrm{m} .
$$

Many typical values for the data have been gathered in [5]. Typical value for mass of a lung is $m=0.5 \mathrm{~kg}$ for a normal male (e.g. [22]); the mass $m$ is not, however, a sensitive parameter as inertial effects do not play a crucial role. A typical value of $S$ can be deduced, in [19], from the inertance $I=m / S^{2}=0.0074 \mathrm{mmHg} \cdot \mathrm{l}^{-1} \cdot \mathrm{s}^{2}=$ $9.8610^{-3} \mathrm{~Pa} \cdot \mathrm{m}^{-1} \cdot \mathrm{s}^{2}$. The value of the resistance $R_{0}=1 \mathrm{mmHg} \cdot \mathrm{s} \cdot \mathrm{l}^{-1}=1.33 \cdot 10^{5} \mathrm{~Pa} \cdot \mathrm{s} \cdot \mathrm{m}^{-3}$ can be found in [27]; By [3], the value of the spring constant is deduced from the typical value of the lung elastance $E=k_{0} / S^{2}=2.5 \mathrm{mmHg} \cdot \mathrm{l}^{-1}=3.3210^{5} \mathrm{~Pa} \cdot \mathrm{s} \cdot \mathrm{m}^{-3}$, so that $S=0.044 \mathrm{~m}^{2}$. The value of $\mu$ is obtained as follows: the resistive forces are due to the set of tissues (20\%) and the bronchial tree (80\%). The influence of the bronchial deformation is neglected in this first description and the external force is the effective force that is imposed by the action of the diaphragm. To say it clearly, it is the control parameter that allows us to reproduce the experimental curves and we will use a time-slot (pulse) external force which is

- positive during (tidal or forced) inspiration,

- equal to 0 during tidal expiration,

- negative during forced expiration.

This control parameter is reproduced on Fig.6. In particular, notice that the deep expiration (resp. inspiration) corresponds to a maximal negative (resp. positive) force $f_{\min }<0$ (resp. $f_{\max }>0$ ). For the proposed choice, we have

$$
f_{\min }=-11 \mathrm{~N}, \quad f_{\max }=13 \mathrm{~N}
$$

and the minimal displacement $x_{\min }$ and maximal displacement $x_{\max }$ have been obtained from experimental curves. 


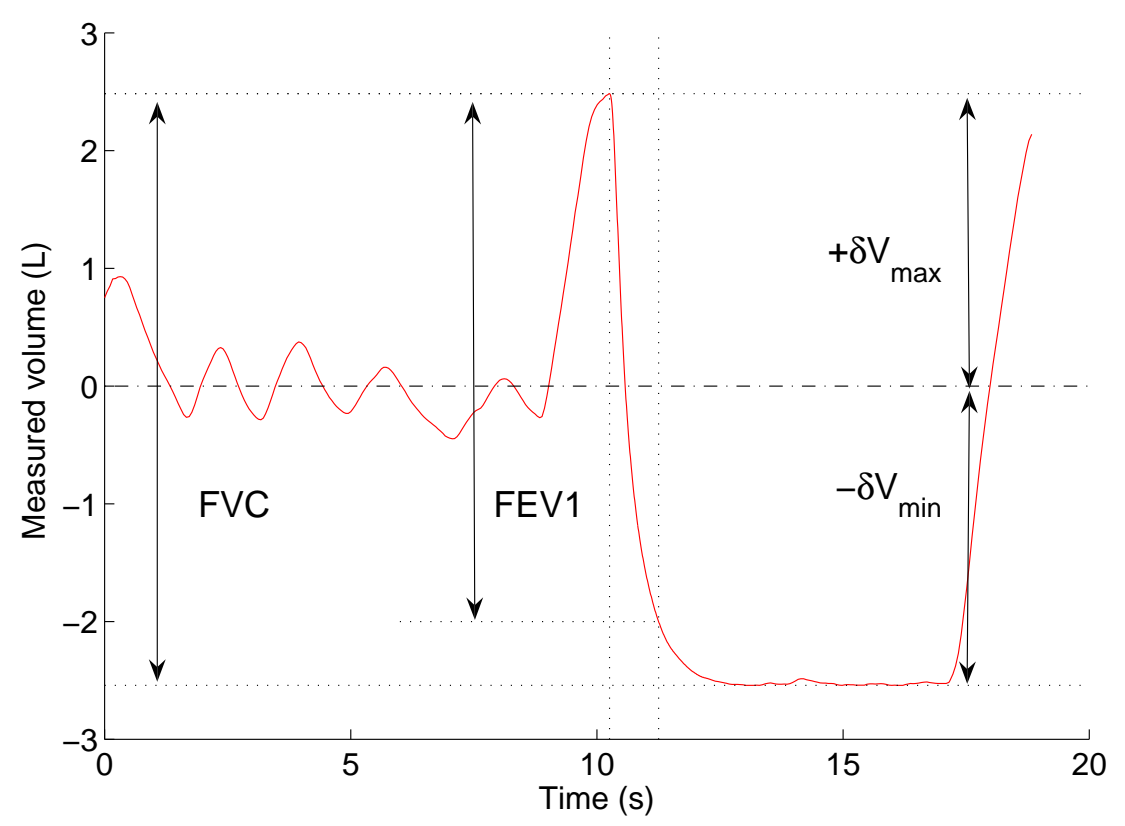

Figure 2. Experimental results: time (in seconds) - volume (in liters) for a normal subject during forced maneuvers: the measured volume corresponds to $\delta V$.

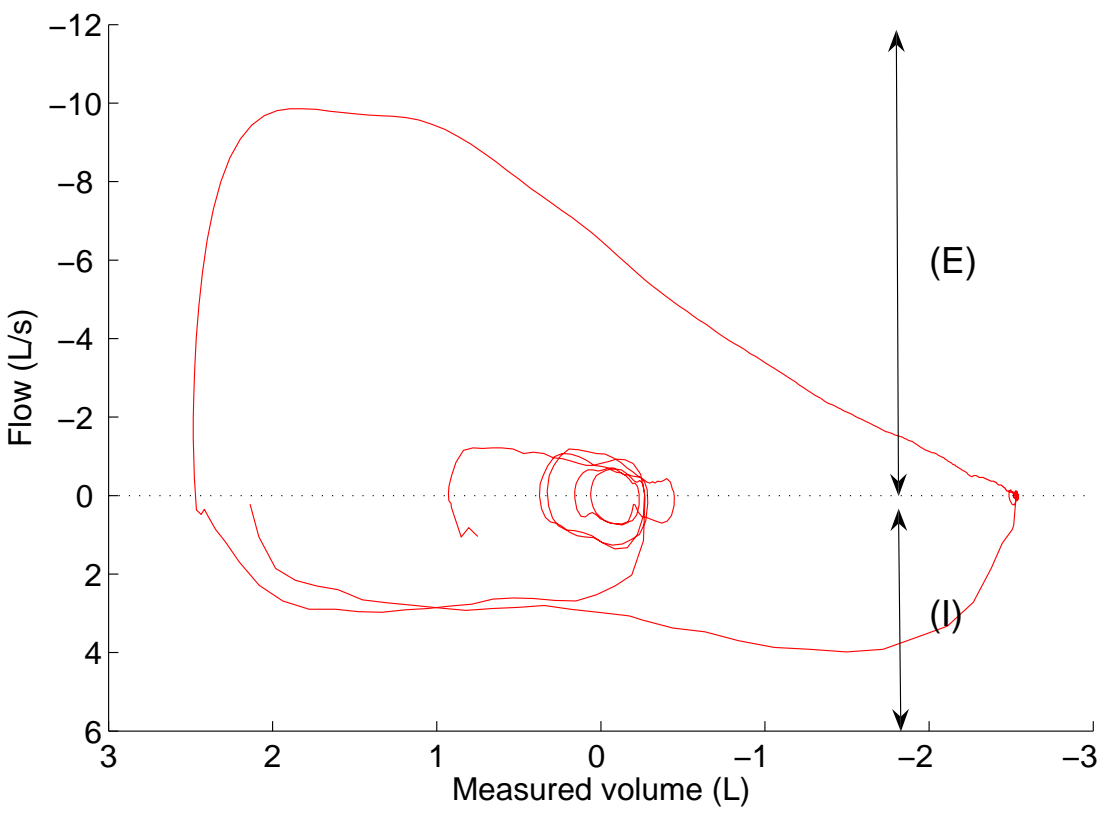

FiguRE 3. Experimental results: volume (in liters) - flow (in liters per second) diagram for a normal subject during forced maneuvers: (E) is the expiratory domain; (I) is the inspiratory domain. 


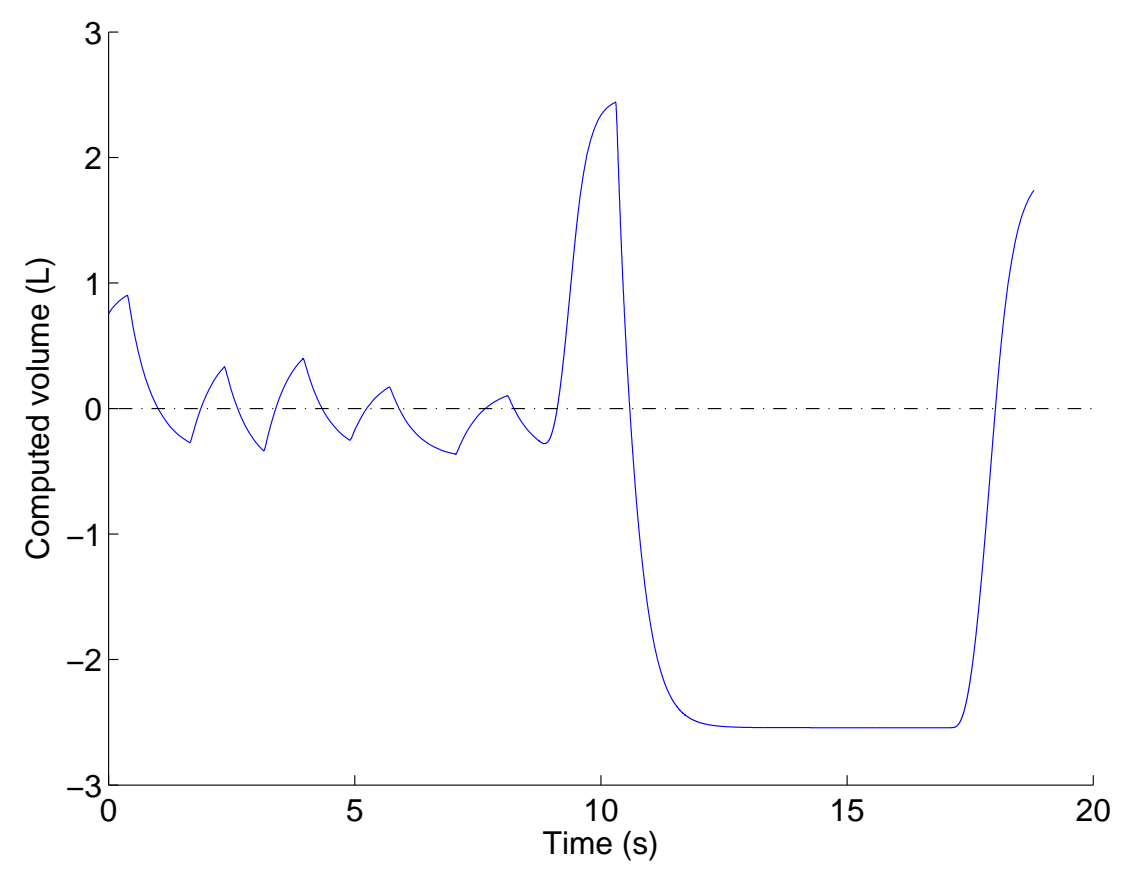

FIGURE 4. Numerical results: time (in seconds) - volume (in liters) for a normal subject during forced maneuvers: the volume variation is computed with the single-compartment model.

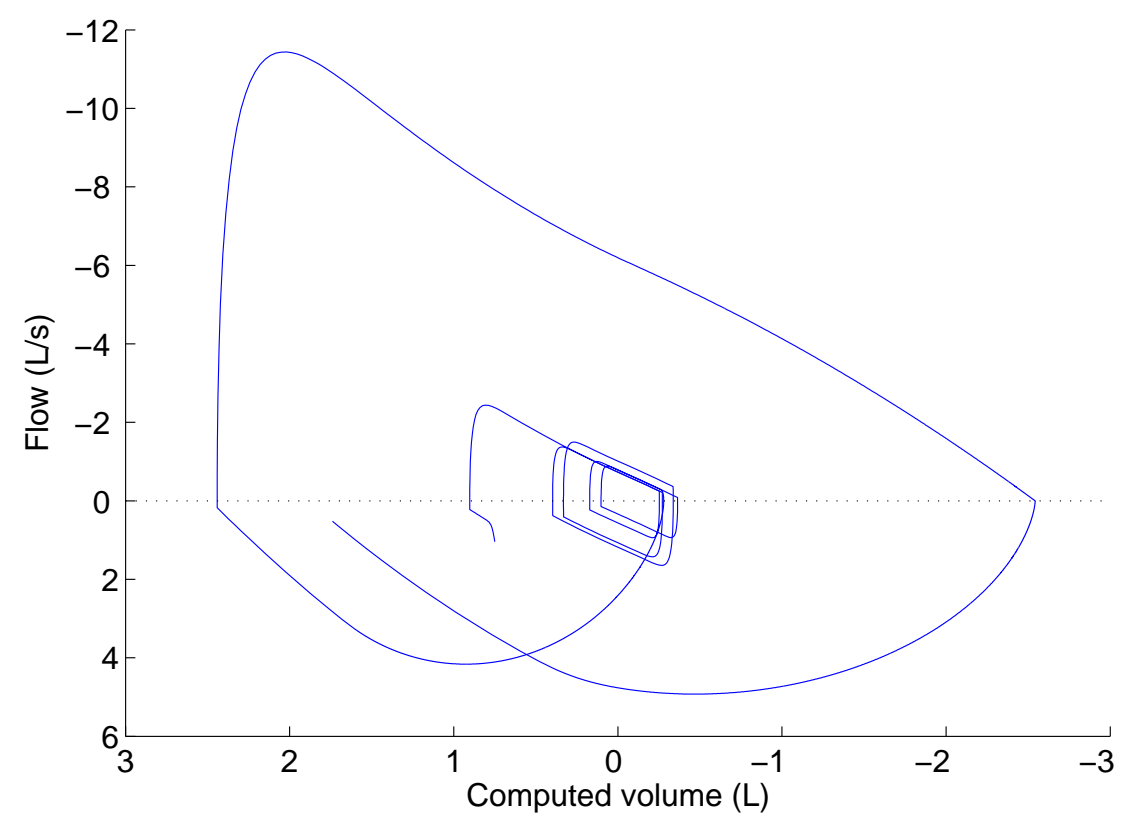

Figure 5. Numerical results: volume (in liters) - flow (in liters per second) diagram for a normal subject during forced maneuvers, computed with the single-compartment model. 


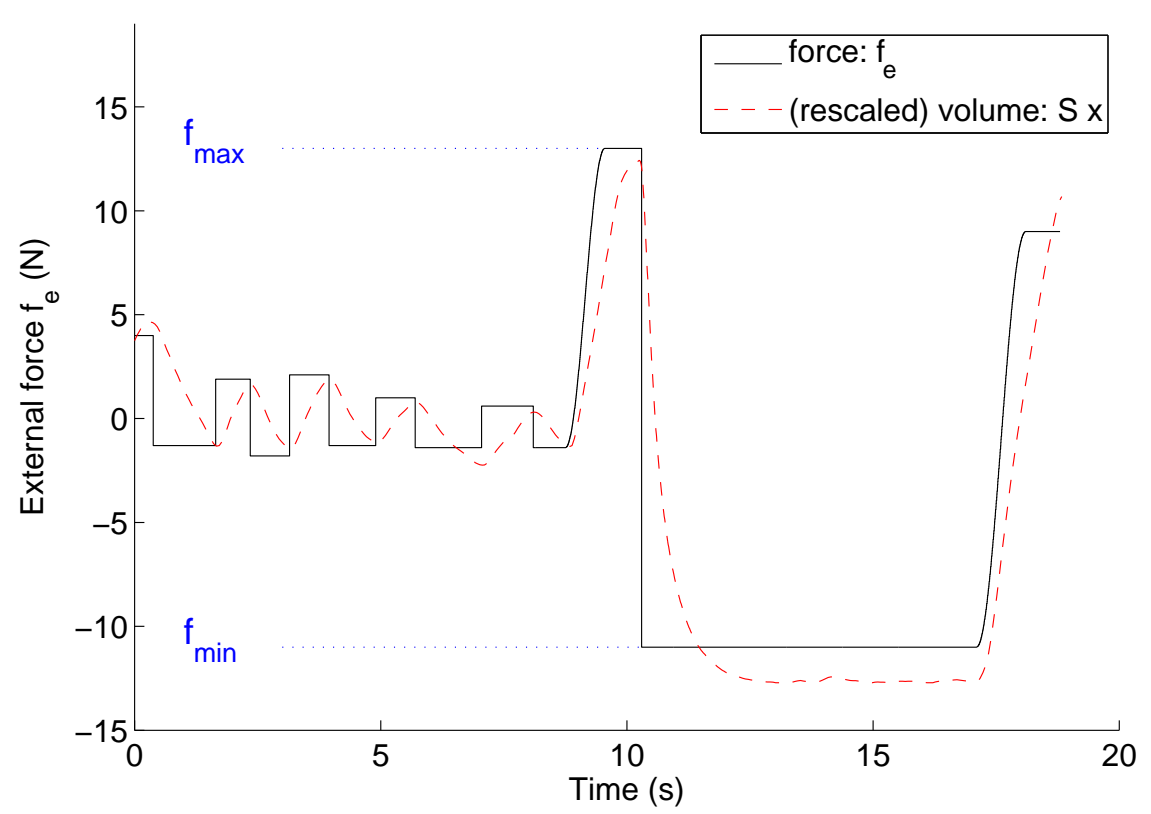

Figure 6. External force $f_{e}$.

The numerical method is based on classical Runge-Kutta methods for stiff problems: in particular, we have used an implementation of TR-BDF2, an implicit Runge-Kutta formula with a first stage that is a trapezoidal rule step and a second stage that is a backward differentiation formula of order 2 . The only parameter that has been controlled is the force term, which has been fitted in order to obtain an good approximation of the experimental curves.

The time-volume curve and volume-flow diagram corresponding to our single-compartment model have been computed with the mentioned data; they are presented on Fig.5. Numerical and experimental results are very similar and allow us to study some qualitative properties of the model. As well, the typical shape of the flow-volume diagram has been numerically reproduced and the peak expiratory flow rates are very close.

In the framework of forced maneuvers, the influence of the bronchial tree stiffness, approximated as bronchial wall compliance, on FEV1/FVC is described by FIG. 7 and shows some qualitative trends.

\section{Gas exchange in the Lung model}

\subsection{Notion of efficiency: variations of the exchange surface area due to the airway wall compliance}

In this subsection, we describe a simple criterion for the definition of efficiency. The principal function of the lung is to transport oxygen from the atmosphere into the bloodstream, and to excrete carbon dioxide from the bloodstream into the atmosphere: thus efficiency of the ventilation process should not rely on the quantity of air that penetrates into the lung but rather on the quantity of oxygen which is brought into the bloodstream. Yet the flux of $\mathrm{O}_{2}$ is directly related to the size of the exchange surface area. This area (under the homothetic deformation assumption), denoted $S_{A}$, can be expressed as a function depending on the volume of the alveoli, i. e.

$$
S_{A} \propto V_{A}^{2 / 3} \propto\left(1+(1-\theta) S x / V_{B}^{0}\right)^{2 / 3}
$$




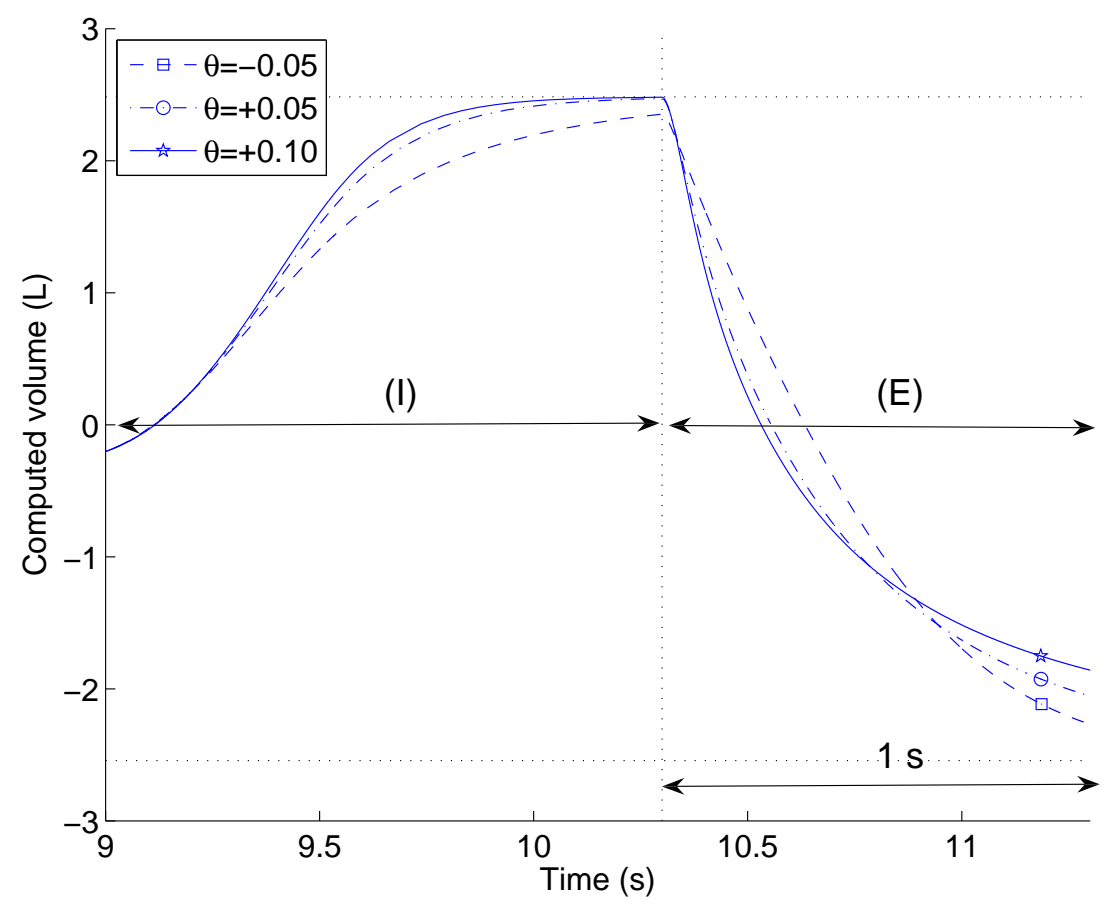

Figure 7. Time (in seconds) - Volume (in liters) for different values of $\theta$.

The first model defining a measure of the efficiency is based on the introduction of the quantity $q$ of $O_{2}$ which has diffused into the bloodstream. This quantity is defined as a time integral of the flux which is proportional to the exchange surface area, as previously mentioned:

$$
q=\int_{0}^{t}\left(1+(1-\theta) S x / V_{B}^{0}\right)^{2 / 3} \mathrm{~d} t .
$$

For a given time (typically equivalent to some respiratory cycles), this model provides an estimate that characterizes the relationship

$$
\theta \mapsto q(\theta),
$$

in which $\theta$ quantifies the intrinsic compliance of the bronchial wall. When $\theta$ is small, the airway is rigid and inspiration has little effect on the volume of the bronchial tree and the resistance of the airway is little affected by the increase in lung volumes. This can for instance be due to the airway smooth muscle tone limiting the "passive" bronchodilation that results from the elastic traction exerted on the bronchi by the surrounding lung parenchyma. As a consequence, the volume of air that can flow across the airway for a given inspiratory driving force is reduced, but the proportion of the inspired air that becomes available at the alveolar volume is increased. This corresponds to an increased exchange surface. On the contrary, when $\theta$ is large, the bronchial tree is more distensible. It dilates under the effects of the inflating forces that are transmitted to it. For a given inspiratory force, more air enters the thorax, but less reaches the alveoli. This corresponds to an increase in what is known as dead space in respiratory physiology, and that is defined as aerated but unperfused regions of the respiratory system. A parametric study is presented in next subsection for a more complex model (which takes into account the $\mathrm{O}_{2}$ impoverishment, as it will be precised further). Notice that a similar study can be 
realized in the framework of the model (2)-(5), which assumes that the partial pressure of $\mathrm{O}_{2}$ is constant: it can be shown that results are very similar, in a normal regime (tidal inspiration / expiration).

\section{2. $\mathrm{O}_{2}$ impoverishment}

We introduce the concentration of $\mathrm{O}_{2}$ in the alveoli, denoted $c$. The total quantity of $\mathrm{O}_{2}$ in the alveoli is $c V_{A}$. We assume that the flux of $\mathrm{O}_{2}$ that passes through the alveolar membranes into the bloodstream, denoted $\dot{q}$, is proportional to the product of the concentration of $\mathrm{O}_{2}$ and the exchange surface area. This area is proportional to $V_{A}^{2 / 3}$, with $V_{A}=V_{A}^{0}+(1-\theta) S x$, so that

$$
\dot{q} \propto\left(V_{A}^{0}+(1-\theta) S x\right)^{2 / 3} c .
$$

Moreover, the quantity of $\mathrm{O}_{2}$ in the alveoli is modified as follows:

(i) The alveoli are supplied with fresh air through the bronchial tree; this flux of $\mathrm{O}_{2}$ is expressed as the product of $\dot{V}_{A}$ and the concentration of $\mathrm{O}_{2}$ at the open boundary of the considered domain (the set of all alveoli) which is either $c_{0}$ (concentration of $\mathrm{O}_{2}$ in fresh air) during inspiration or $c$ during expiration. Using the notation

$$
[y \geq 0 ? a, b]= \begin{cases}a & \text { if } y \geq 0 \\ b & \text { else }\end{cases}
$$

Thus the supplied flux of $O_{2}$ is $\dot{V}_{A}\left[\dot{x} \geq 0 ? c_{0}, c\right]$.

(ii) At the alveolar membrane, $\mathrm{O}_{2}$ diffuses into the blood. As it was mentioned the corresponding flux is $\dot{q}$. The variation of the total quantity of $\mathrm{O}_{2}$ in the alveoli satisfies:

$$
\dot{c}=\frac{1}{V_{A}}\left(\dot{V}_{A}\left[\dot{x} \geq 0 ? c_{0}-c, 0\right]-\dot{q}\right) .
$$

Thus, the respiratory system model including the influence of the bronchial wall compliance and $\mathrm{O}_{2}$ impoverishment can be written as a first order O.D.E.:

$$
(\mathrm{RS})\left\{\begin{aligned}
\dot{x} & =u \\
\dot{u} & =\frac{1}{m}\left(f_{e}-\left(\frac{R_{0} S^{2}}{1+\theta S x / V_{B}^{0}}+\mu\right) u-k x\right) \\
\dot{q} & =\Lambda\left(V_{A}^{0}+(1-\theta) S x\right)^{2 / 3} c \\
\dot{c} & =\frac{1}{V_{A}}\left(\dot{V}_{A}\left(c_{0}-c\right) \mathbf{1}_{\mathbb{R}^{+}}(u)-\dot{q}\right) .
\end{aligned}\right.
$$

Notice that the constant $\Lambda$ which quantifies the trend of $O_{2}$ to be diffused through the alveolar membranes into blood, plays an important role: it is directly involved in the characteristic time of $\mathrm{O}_{2}$ apoverishment. In a normal regime, when considering a situation without incoming flux of fresh air, in the vinicity of $x_{\max }$, we have $\dot{q}=-V_{A} \dot{c}$ so that the following equation holds:

$$
\dot{c}=-\lambda c, \quad \lambda:=\lambda(x)=\Lambda\left(V_{A}^{0}+(1-\theta) S x\right)^{-1 / 3} .
$$

Here, $\lambda^{-1}$ is the characteristic time for decay of $O_{2}$ and it is proportional to $\Lambda^{-1}$. The value of $\Lambda$ can be computed as follows: the typical value of $\lambda^{-1}$ in a static regime, the rib cage being swelled at most, is about 5 seconds. Thus, $\Lambda$ will be defined as $\left(V_{A}^{0}+(1-\theta) S x_{\max }\right)^{1 / 3} / \lambda^{-1}$. For small values of $\theta$, i. e. $\theta \approx 0-0.2$, then $\Lambda$ is of order $0.025 \mathrm{~m} \cdot \mathrm{s}^{-1}$.

The enrichment of the model does not alter the behaviour of the model, in a normal regime (for which the concentration of $\mathrm{O}_{2}$ does not decrease significantly) but, in case when the resistance is high, it is important to notice that the air in alveoli is only partially renewed: this leads to a sharp drop in partial pressure of $O_{2}$ in the alveoli and, consequently, efficiency of the process. 


\subsection{Numerical results: influence of the bronchial wall compliance}

In this subsection, we have considered system $(\mathrm{RS})$ with the following initial conditions:

$$
x=0, \quad u=0, \quad q=0, \quad c=c_{0} .
$$

The external force $f_{e}$ is defined on some time interval $[0, \tau]$ as

$$
f_{e}(t)= \begin{cases}F, & \text { if } t \in[0, \eta \tau[ \\ 0, & \text { if } t \in[\eta \tau, \tau[\end{cases}
$$

and this signal is then $\tau$-periodically reproduced. In particular, this means that the expiration is unforced (tidal regime) and also that $\eta \in[0,1]$ denotes the fractional rate of inspiration time during one inspiration-expiration cycle. We have used the following data:

$$
\begin{gathered}
m=0.25 \mathrm{~kg}, \quad S=0.010 \mathrm{~m}^{2}, \quad R=3 \cdot 10^{5} \mathrm{~Pa} \cdot \mathrm{s} \cdot \mathrm{m}^{-3}, \quad E=1 \cdot 10^{5} \mathrm{~Pa} \cdot \mathrm{s} \cdot \mathrm{m}^{-3}, \quad k=E S^{2}, \quad \mu=0.25 R S^{2}, \\
V_{A}^{0}=0.2 \cdot 10^{-3} \mathrm{~m}^{3}, \quad V_{B}^{0}=0.3 \cdot 10^{-3} \mathrm{~m}^{3}, \quad \tau=6 \mathrm{~s}, \quad \eta=0.35, \quad F=3 \mathrm{~N}, \quad \Lambda=0.025 \mathrm{~m} \cdot \mathrm{s}^{-1}, \quad c_{0}=1 .
\end{gathered}
$$

The choice for the value of $\theta$ (role of the airway wall compliance) and $\eta$ (importance of the inspiratory process) will be discussed further. The problem of how to relate some maximal efficiency to an optimal parameter is questioned in the following numerical tests. Notice that these studies allow us to introduce some qualitative trends with respect to some parameters; nevertheless, numerical results appear to be very sensitive with respect to the physiological data so that the lack of precision on these data prevents us from providing quantitative results.

We focus on the role of the bronchial wall compliance with respect to the efficiency criterion. Choosing $\eta=0.35$, we study the influence of $\theta$ on the $\mathrm{O}_{2}$ quantity, denoted $q$. The results have been led by means of an integration process over 20 cycles and they are presented on Fig. 8: negative values of $\theta$ model in a simple way some kind of an hyperactivity of the smooth muscle (i. e. during inspiration, the size of the bronchi decreases). At inspiration, when $\theta$ is close to 0 , the volume of the bronchi almost does not vary: on one hand, the resistance decreases only a little (i. e. less air is driven in the airways) but, on the other hand, the increase in thoracic volume occurs mainly in the alveolar compartment, which tends to increase the exchange surface area. Therefore, we have a competition between two antagonist effects and we may define a maximal efficiency for some value of $\theta$. Firstly, it is observed that maximal efficiency is attained with $\theta_{\text {opt. }}=0.11$. Secondly, the efficiency falls down suddenly when starting from this optimal value and decreasing the value of $\theta$, meaning that the system is rather unstable on the left of the optimal value. Notice that $\theta_{\text {opt. }}$ widely differs from the value $\theta_{0}$ corresponding to a similar deformation of bronchi and alveoli:

$$
\theta_{0}=\frac{V_{A}^{0}}{V_{A}^{0}+V_{B}^{0}}=0.4
$$

Thus, the case $\theta=\theta_{\text {opt. }}$ corresponds to little deformation of the bronchi and, as pointed out in Remark 2.1, highlights the active role of the bronchial wall compliance as an important feature of the lung efficiency. Finally Fig. 9 provides the numerical results obtained with the optimal parameter.

Note that the efficiency curve (shown on FIG. 8) exhibits a stiff decrease on the left of the optimal point. The model reproduces the well-known fact that an increase of bronchial rigidity from an already high starting value has much greater consequences upon the ventilation efficiency than a perturbation starting from a standard value (healthy situation). From this point of view, the optimal configuration $(\theta=0.11$ in our situation) can be considered dangerous because it is quite sensitive to small perturbations. This situation is in line with the conclusions of [15], where the authors investigate the notion of geometric optimality of the bronchial tree with regard to the global resistance under a volume constraint. Suboptimality with respect to this resistance 


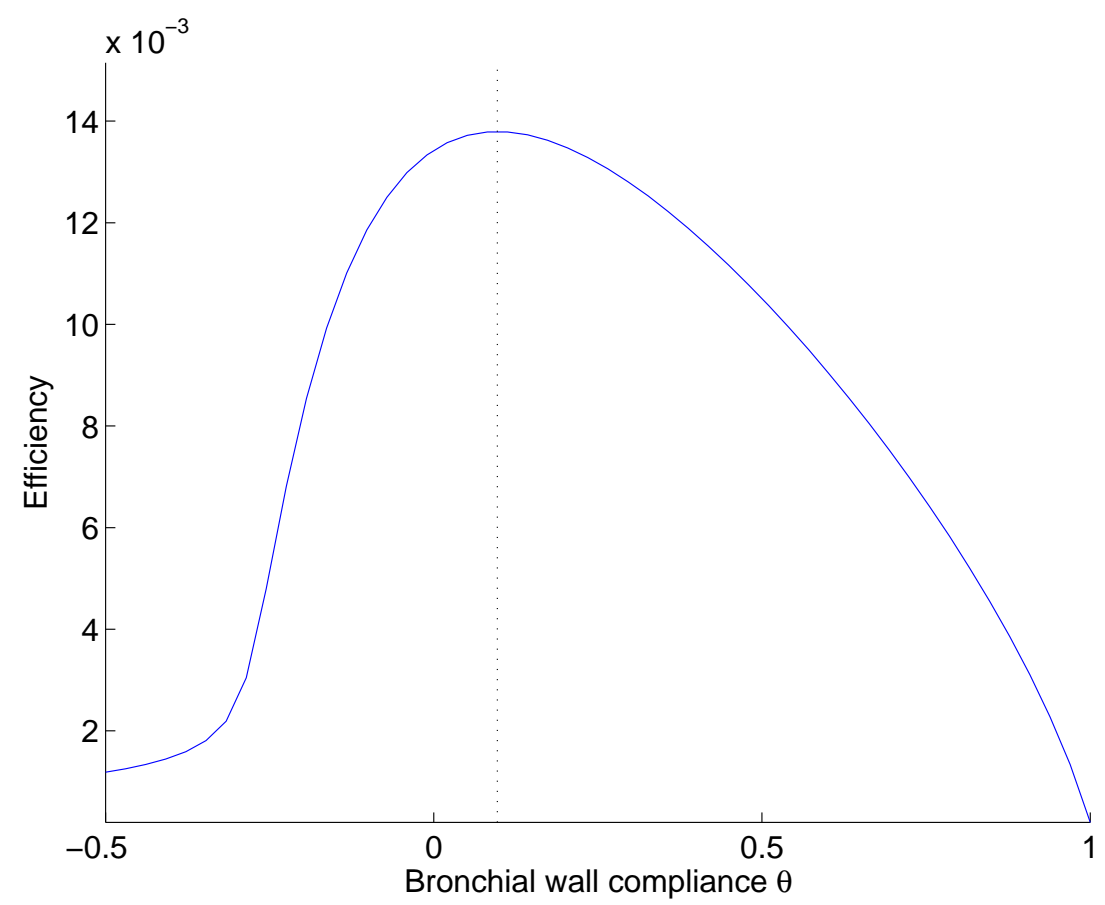

FiguRE 8. Influence of bronchial wall compliance on the efficiency number: graph of $\theta \mapsto q(\theta)$.
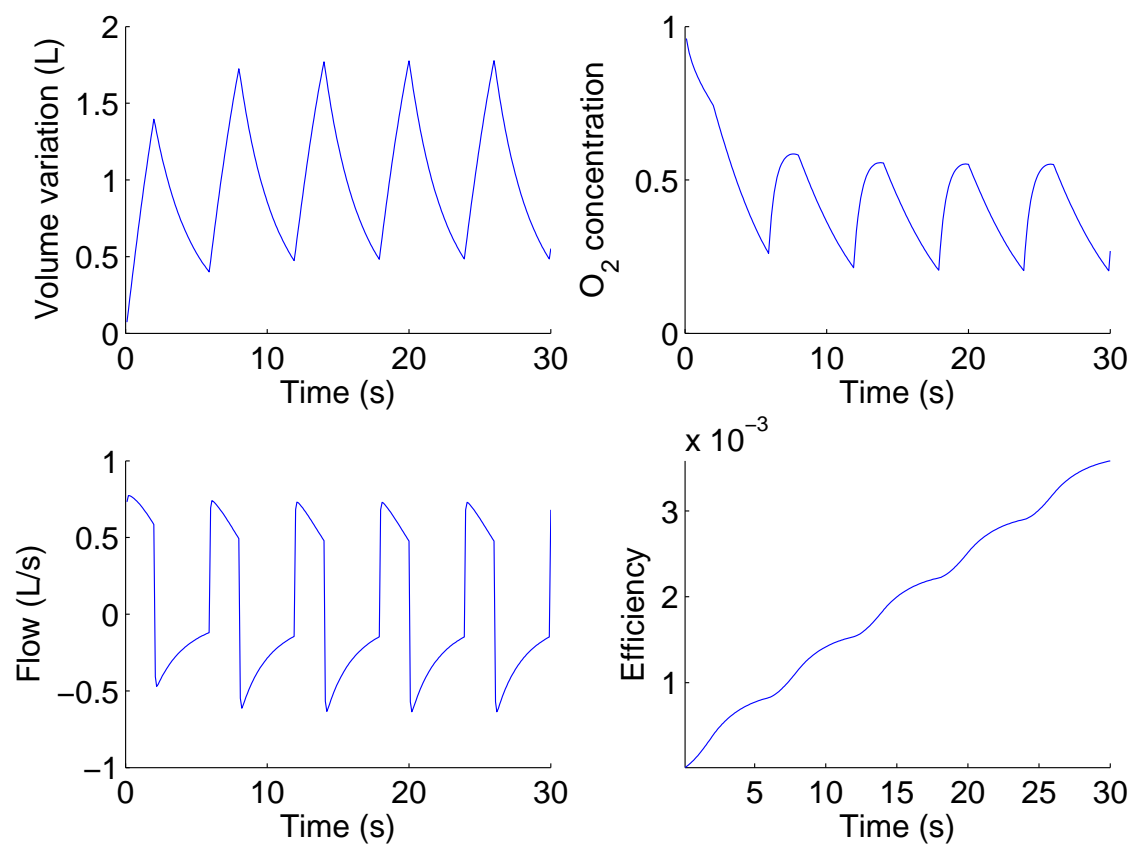

Figure 9. Displacement, flow, normalized $\mathrm{O}_{2}$ concentration in the alveoli and efficiency number which has been brought into the bloodstream for the optimal choice of $\theta$, i. e. $\theta_{\text {opt. }}=0.11$. 
criterium, which is observed in practice, appears to be a compromise between optimality and robustness with respect to perturbations.

\section{Conclusion}

In conclusion, the combination of a single compartment model of the respiratory system that is accurate enough to predict the behaviour of the system during a complex dynamical situation (the forced maneuver), on one hand, and of an oxygen absorption model, on the other hand, strongly suggests that there is a relationship between respiratory mechanics parameters and the efficiency of the lungs as gas exchangers. The model shows that the mechanical response of the bronchial tree to the dilating forces related to inspiration is among the determinants of this efficiency. At one extreme, a fully rigid bronchial tree would be counterproductive by preventing sufficient amounts of gas to flow through it and reach the alveoli. At the other extreme, a fully distensible bronchial tree would allow greater inspiratory volumes to enter the thorax, but would compromise the gas exchange efficiency by preventing the alveoli to "benefit" from the inhaled volume. The distensibility of the proximal part of the bronchial tree is limited by its cartilaginous armature. Beyond the 14-15th generation of divisions of the bronchial tree, namely at a point where the bronchial diameter is of about 1-2 mm, this cartilaginous structure lacks. This is bound to make the bronchial tree highly distensible, and yet at this point they are three-dimensionally surrounded by the alveoli and hence intensely submitted to elastic forces. In the absence of any armature, the inspiratory related dilating forces would provoke a major increase in the bronchial volume with the deleterious effects on the alveolar volume that are predicted by our model. One possible function of the airway smooth muscle could be to prevent this phenomenon by stiffening the airway wall. This would be the result of the structural rigidity merely provided by the intrinsic mechanical properities of ASM rings or strips, and also of the bronchial smooth muscle tone. One function of the airway smooth muscle would thus be to provide a mechanical compromise between fully rigid bronchi and fully distensible ones and hence an optimized transport of the inspired air towards the alveoli, with, furthermore, possible regulations through the sympathetic-parasympathetic control of the ASM tone. In line with this contention, the administration of bronchodilators to normal humans and asthmatics has long been known to increase deadspace (e.g. [13]). This could, among other factors, contribute to explain the decreased arterial oxygen partial pressures that are at times observed after the administration of bronchodilators in asthmatics.

\section{REFERENCES}

[1] S. Abboud, O. Barnea, A. Guber, N. Narkiss and I. Bruderman, Maximum expiratory flow-volume curve: mathematical model and experimental results, Med. Eng. Phys. 17 (1995), no 5, 332-336.

[2] V. Antonaglia, L. Torelli, W.A. Zin and A. Gullo, Effects of viscoelasticity on volume distribution in a two-comportmental model of normal and sick lungs, Physiol. Meas. 26 (2005), 13-28.

[3] R. Begin, A.D. Renzetti Jr., A.H. Bigler and S. Watanabe, Flow and age dependence of airway closure and dynamic compliance, J. Appl. Physiol. 38 (1975), no 2, 199-207.

[4] W. Benish, P. Harper, J. Ward and J. Popovich, Jr., A mathematical model of lung static pressure-volume relationships: comparison of clinically derived parameters of elasticity, Henry Ford Hosp. Med. J. 36 (1988), no 1, 44-47.

[5] A. Ben-Tal, Simplified models for gas exchange in the human lungs, J. Theor. Biol. 238 (2006), 474-495.

[6] J.H. Bates, M.S. Ludwig, P.D. Sly, K. Brown, J.G. Martin and J.J. Fredberg, Interrupter resistance elucidated by alveolar pressure measurement in open-chest normal dogs, J. Appl. Physiol. 65 (1988), 408-414.

[7] J.M. Bogaard, S.E. Overbeek, A.F.M. Verbraak, C. Vons, H.T.M. Folgering, Th.W. van der Mark, C.M. Roos, P.J. Sterk and the Dutch CNSLD study group, Pressure-volume analysis of the lung with an exponential and linear exponential model in asthma and COPD, Eur. Respir. J. 8 (1995), 1525-1531.

[8] G.P.S. Crooke, J.D. Head and J.J. Marini, A general two-compartment model for mechanical ventilation, Math. Comput. Modelling 24 (1996), no. 7, 1-18.

[9] Y.H. Chang and C.P. Yu, A model of ventilation distribution in the human lung, Aer. Sci. Tech. 30 (1999), 309-319.

[10] C. Grandmont, Y. Maday and B. Maury, A multiscale / multimodel approach of the respiration tree, Proceedings of the International Conference "New Trends in Continuum Mechanics" 8-12 September 2003, Constantza, Romania Theta Foundation Publications, Bucharest, 2005.

[11] C. Grandmont, B. Maury and N. Meunier, A viscoelastic model with non-local damping application to the human lungs, Math. Mod. Numer. Anal. 40 (2006), no 1, 201-224. 
[12] D. Grebenkov, M. Filoche and B. Sapoval, Diffusion-reaction in branched structures: theory and application to the lung, Phys. Rev. Lett. 94 (2005), 050602.

[13] R.J. Knudson and H.P. Constantine, An effect of isoproterenol on ventilation-perfusion in asthmatic versus normal subjects, J. Appl. Physiol. 22 (1967), 402-406.

[14] J. Kaye, F.P. Primiano Jr. and D. Metaxas, Anatomical and physiological simulation for respiratory mechanics, J. Img. Guid. Surg. 1 (1995), no 3, 164-171.

[15] B. Mauroy, M. Filoche, J.S. Andrade Jr. and B. Sapoval, Interplay between flow distribution and geometry in an airway tree, Phys. Rev. Lett. 90, 14 (2003).

[16] J. Mead, Contribution of compliance of airways to frequency dependent behaviour of lungs, J. Appl. Physiol. 26 (1969), 670-673.

[17] A.B. Otis, V.B. Mc Kerrow, R.A. Bartlett, J. Mead, M.B. Mc Ilroy, N.J. Selverstone and E.P. Radford, Mechanical factors in the distribution of pulmonary ventilation, J. Appl. Physiol. 8 (1956), 427-443.

[18] Mathematical models in human physiology, J.T. Ottesen, M.S. Olufsen, J.K. Larsen (editors), SIAM, Philadelphia, 2004.

[19] J.R. Rodarte and K. Rehder, Dynamic of respiration, In: Handbook of physiology: the respiratory system, A.P. Fishman, P.T. Macklem, J. Mead and S.R. Geiger (editors), Americal Physiological Society, 131-144, 1986.

[20] T. Similowski and J.H.T. Bates, Two-compartment modelling of respiratory system mechanics at low frequencies: gas redistribution or tissue rheology?, Eur. Respir. J. 4 (1991), 353-358.

[21] S. Tomioka, J.H.T. Bates and C.G. Irvin, Airway and tissue mechanics in a murine model of asthma: alveolar capsule vs. forced oscillations, J. Appl. Physiol. 93 (2002), 263-270.

[22] S.P. Tomlinson, D.G. Tilley and C.R. Burrows, Computer simulation of the human breathing process, IIIE Eng. Med. Biol. Mag., 13 (1994), no 1, 115-124.

[23] V. Thomas, F. Costes and T. Busso, Estimation of arterial $P_{\mathrm{CO}_{2}}$ from a lung model during ramp exercise in healthy young subjects, Resp. Physiol. Neurobiol. 156 (2007), 259-265.

[24] J.G. Venegas, R.S. Harris and B.A. Simon, A comprehensive equation for the pulmonary pressure-volume curve, J. Appl. Physiol. 81 (1998), no 1, 389-395.

[25] E.R. Weibel, Morphometry of the human lung, Springer Verlag and Academic Press, Berlin, New York, 151 pp., 1963.

[26] E.R. Weibel, B. Sapoval and M. Filoche, Design of peripheral airways for efficient gas exchange, Resp. Phys. and Neur. 148 (2005), 3-21.

[27] J.B. West, Respiratory Physiology - The Essentials, Baltimore: Williams \& Wilkins, 1974.

[28] The lung: scientific foundations, R.G. Crystal, J.B. West, E.R. Weibel and P.J. Barnes (editors), 2 Vol., 2nd Edition, Lippincott-Raven Press, Philadelphia, 1997. 